\title{
画HAD
}

DOI: http://doi.org/10.22585/hospdomic.v4i3.109

\section{Calidad de vida de las personas adultas con nutrición parenteral ingresadas en hospitalización domiciliaria: revisión sistemática}

\section{Quality of life of adult people with parenteral nutrition admitted to home hospitalization: systematic review}

Rocío Martínez-González', Javier Sanz-Valero 2,3, Carmina Wanden-Berghe 4,5

1. Universidad Miguel Hernández. Facultad de Farmacia, Campus de Sant Joan d'Alacant. Alicante. España.

2. Instituto de salud Carlos III. Escuela nacional de medicina del Trabajo. Madrid. España.

3. Universidad Miguel Hernández. Departamento de Salud Pública e Historia de la Ciencia, Campus de Sant Joan d'Alacant. Alicante. España.

4. Instituto de Investigación Sanitaria y Biomédica de Alicante (ISABIAL-FISABIO). Alicante. España.

5. Hospital General Universitario de Alicante. Unidad de Hospitalización a Domicilio. Alicante. España.

Correspondencia/Correspondence

Javier Sanz-Valero

Email: fi.sanz@isciii.es

Recibido/Received

01.06 .2020

Aceptado/Accepted

23.06.2020
Conflicto de Intereses/Competing interest Los autores y autora del trabajo declaran no tener ningún conflicto de interés 


\section{RESUMEN}

Objetivo: Revisar la documentación científica relacionada con la calidad de vida de las personas adultas con nutrición parenteral ingresadas en hospitalización domiciliaria.

Método: Revisión crítica y sistemática. Los datos se obtuvieron de la consulta a las siguientes bases de datos bibliográficas: MEDLINE (vía PubMed), Cochrane Library, Embase, Scopus y Web of Science. Los términos utilizados, como descriptores y como texto en los campos de registro del título y el resumen, fueron "Home Care Services", "Parenteral Nutrition" y "Quality of Life", utilizando los filtros «Humans», «Adult: 19+ years» y «Clinical Trial». Fecha de la búsqueda febrero de 2020. La calidad documental de los artículos se evaluó mediante el cuestionario CONSORT.

Resultados: De las 379 referencias recuperadas, tras depurar las repeticiones y aplicar los criterios de inclusión y exclusión, se seleccionaron 7 ensayos clínicos. En 3 (43\%) de los ensayos revisados se observó una mejora en relación a la calidad de vida. Las puntuaciones obtenidas mediante el cuestionario CONSORT, oscilaron entre 12,5 y 20,5 sobre una puntuación máxima de 24.

Conclusiones: El incremento de la calidad de vida de los enfermos con NPD está directamente relacionado con la del estado y soporte nutricional de los mismos. Se observó una mejor calidad de vida en los pacientes con bomba portátil y en los que se administró teduglutida. El adecuado manejo del catéter y la consecuente disminución de las infecciones también contribuyó a la mejora de la calidad de vida de las personas con NPD.

Palabras clave: Nutrición Parenteral; Calidad de Vida; Servicios de Atención de Salud a Domicilio; Apoyo Nutricional.

\section{ABSTRACT}

Objective: To review the scientific documentation related to the quality of life of adult people with parenteral nutrition admitted to home hospitalization.

Method: Critical and systematic review. The data were obtained from the consultation of the following bibliographic databases: MEDLINE (via PubMed), Cochrane Library, Embase, Scopus y Web of Science. Terms used as descriptors and as text in the title and summary record fields were: "Home Care Services", "Parenteral Nutrition" and "Quality of Life", using the filters «Humans», «Adult: 19+ years» y «Clinical Trial». Search date February 2020. The documental quality of the articles was evaluated by using the CONSORT questionnaire.

Results: From 379 recovered references, after refining the repetitions and applying the inclusion and exclusion criteria, 7 clinical trials were selected. In 3 (43\%) of the revised trials it was observed an improvement in relation to the quality of life. The scores obtained by the CONSORT questionnaire were from 12.5 to 20.5 with a maximum score of 24 .

Conclusions: The parenteral support of patients with home parenteral nutrition (HPN) was directly related to the nutritional status, and it corresponded with an increase in quality of life. An improvement of quality of life was proved in patients that used portable pump and it was likewise proved that the use of teduglutide was beneficial for the quality of life. The correct handle of the catheter and the reduction of infections were also connected with the enhancement of the quality of life of people with HPN.

Keywords: Parenteral Nutrition; Quality of Life; Home Care Services; Nutritional Support. 


\section{INTRODUCCIÓN}

El concepto de calidad de vida relacionado con la salud (CVRS) se contextualizó en relación a como la enfermedad (como fuente de dolor, disfunción física e incomodidad) imponía limitaciones o alteraciones en el comportamiento diario, actividades sociales y bienestar psicológico, así como en otros aspectos de vida cotidiana (1).

La medición de la calidad de vida aporta una dimensión holística al curso del estado clínico o a la respuesta a una intervención determinada. Además, medir la CVRS es un proceso complejo que responde a las expectativas individuales en diferentes facetas de la vida. La forma en que la CVRS se ve afectada por el estado nutricional del paciente es un tema de constante interés y debate permanente (2). Es conocido, que un empobrecimiento del estado nutricional conduce a una disminución de la función fisiológica, aumentando el riesgo de complicaciones y muerte séptica, situación que se ve agravada con la edad $(3,4)$. Además, existe una correlación significativa entre la nutrición y las alteraciones musculares, inmunes y cognitivas y, por lo tanto, una mejora en el estado nutricional es un factor importante en la mejora de la función fisiológica (5).

Por otro lado, la hospitalización a domicilio (HaD) aporta ventajas como otra modalidad de hospitalización distinta a la convencional. Existe una contrastada evidencia acerca de dichas ventajas en lo que respecta a la eficacia, seguridad y al alto nivel de satisfacción de esta modalidad asistencial. Los factores clave para su correcta implementación son garantizar una adecuada selección de los pacientes dependientes de $\mathrm{HaD}$ y disponer de un equipo experimentado en atención de casos complejos en el domicilio (6).

Así, la $\mathrm{HaD}$ se define como un servicio que proporciona tratamiento activo por profesionales de la salud en el hogar del paciente, por una afección que de otro modo requeriría atención hospitalaria. La implementación de los servicios hospitalarios en el hogar tiene también como objetivo mejorar la percepción del paciente respecto al tratamiento y mejorar su calidad de vida al ofrecer la posibilidad de dicha atención en un ambiente amigable y con la misma eficiencia en la atención médica (7).

La nutrición parenteral domiciliaria (NPD) es una alternativa terapéutica para los pacientes que necesitan alimentarse por vía parenteral para poder vivir. Consiste en la administración de soluciones a través de accesos venosos de larga duración, en el propio domicilio del enfermo, en pacientes en los que no se puede conseguir un adecuado aporte calórico-proteico por vía digestiva. No obstante, la NPD es un proceso de logística compleja en el que intervienen diversos especialistas sanitarios, así como el propio paciente, su familia o su cuidador $(8,9)$. En consecuencia, los pacientes con NPD enfrentan, en ocasiones, complicaciones que pueden influir en su vida personal y, por tanto, en la CVRS (10).

Así pues, el objetivo de este estudio fue revisar la documentación científica relacionada con la calidad de vida de las personas adultas con nutrición parenteral ingresadas en hospitalización domiciliaria. 


\section{MÉTODOS}

\section{Diseño}

Estudio descriptivo transversal y análisis crítico de los trabajos recuperados mediante revisión sistemática.

\section{Fuente de obtención de los datos}

Los datos se obtuvieron de la consulta directa y acceso, vía Internet, a las siguientes bases de datos bibliográficas del ámbito de las ciencias de la salud: MEDLINE (vía PubMed), Cochrane Library, Embase, Scopus y Web of Science.

\section{Tratamiento de la información}

Para definir los términos de la búsqueda se consultó el Thesaurus desarrollado por la U.S. National Library of Medicine. Se consideró adecuado el uso de los términos "Home Care Services", "Parenteral Nutrition" y "Quality of Life", como descriptores y como texto en los campos de registro del título y el resumen, conformando la sintaxis de búsqueda final mediante la intersección booleana de tres ecuaciones: (Población) AND (Intervención) AND (Resultado).

- Población - Personas adultas atendidas por los servicios de cuidado domiciliario:

"Home Care Services"[MeSH] OR "Home Care"[Title/Abstract] OR "Domiciliary Care"[Title/ Abstract] OR "Home Care Services, Hospital- Based"[Mesh] OR "Hospital-Based Home Care"[All Fields] OR "Hospital Based Home Care"[Title/Abstract] OR "Hospital Home Care Services"[Title/ Abstract] OR "Hospital-Based Home Care Services"[Title/Abstract] OR "Hospital Based Home Care Services"[Title/Abstract] OR "Home Hospitalization"[Title/Abstract] OR "Hospital at Home"[Title/Abstract] OR "Hospital-at-Home"[Title/Abstract] OR "Hospital Home Care"[Title/Abstract] OR "Hospital at Home Care"[Title/Abstract] OR "Hospital in the Home"[Title/Abstract]

- Intervención - Nutrición Parenteral:

"Parenteral Nutrition"[Mesh] OR "Parenteral Nutrition"[Title/Abstract] OR "Parenteral Feeding"[Title/Abstract] OR "Intravenous Feeding"[Title/Abstract] OR "Parenteral Alimentation"[Title/ Abstract] OR "Hyperalimentation Parenteral"[Title/Abstract] OR "Parenteral Hyperalimentation"[Title/ Abstract]

- Resultado - Calidad de Vida:

"Quality of Life"[Mesh] OR "Quality of Life"[Title/Abstract] OR "Life Quality"[Title/Abstract] OR "Health Related Quality Of Life"[Title/Abstract] OR "HRQOL"[Title/Abstract]

La ecuación de búsqueda final se desarrolló para su empleo en la base de datos MEDLINE, vía PubMed, utilizando los filtros: «Humans», "Adult: 19+ years» y «Clinical Trial».

Esta estrategia se adaptó a las características de cada una del resto de bases de datos consultadas. La búsqueda se realizó desde la primera fecha disponible, de acuerdo a las características de cada base de datos, hasta febrero de 2020 y se completó con el examen del listado bibliográfico de los artículos que fueron seleccionados.

\section{Selección final de los artículos}

Se escogieron para la revisión y análisis crítico los artículos que cumplieron los siguientes criterios: adecuarse a los objetivos de la búsqueda (calidad de vida de pacientes con nutrición paren- 
teral en el domicilio), estar publicados en revistas arbitradas por pares y escritos en inglés, español o portugués.

La selección de los artículos pertinentes se realizó de forma independiente por los autores: RMG y JSV. Para dar por válida la inclusión de los estudios se estableció que la valoración de la concordancia entre estos autores (índice Kappa) debía ser superior al 60\% (fuerza de la concordancia buena). Siempre que se cumpliera esta condición, las posibles discordancias se solucionaron mediante el consenso entre todos los autores.

Para valorar la calidad de los documentos seleccionados se utilizaron las directrices para la comunicación de los ensayos clínicos CONSORT (CONsolidated Standards of Reporting Trials) (11), que contiene un listado de 25 aspectos esenciales que deben describirse en la publicación de estos estudios. Para cada artículo seleccionado se asignó un punto por cada ítem presente (en caso de no ser aplicable no puntuaba). Cuando un ítem estaba compuesto por varios puntos, estos se evaluaron de forma independiente, dándole el mismo valor a cada uno de ellos y posteriormente se realizó un promedio (siendo éste el resultado final de ese ítem), de tal forma que en ningún caso se pudiera superar la puntuación de un punto por ítem.

Para conocer el nivel de evidencia y su grado de recomendación se utilizaron las recomendaciones de la U. S. Agency for Healthcare Research and Quality (AHRQ) (12).

\section{Extracción de los datos}

El control de la corrección de los datos se realizó mediante dobles tablas que permitieron la detección de las desviaciones y su subsanación mediante nueva consulta de los originales.

Para determinar la actualidad de los artículos se calculó el semiperíodo de Burton-Kebler (la mediana de la edad) y el Índice de Price (porcentaje de artículo con edad inferior a los 5 años).

Los estudios se agruparon según las variables a estudio, con el fin de sistematizar y facilitar la comprensión de los resultados, considerando los siguientes datos: primer autor y año de publicación, diseño, población incluida en los artículos revisados, patología de la población, país, periodo del estudio tratamiento, tipo de nutrición, forma y frecuencia de administración, método de evaluación de la calidad de vida y resultados obtenidos de la intervención.

\section{RESULTADOS}

Tras aplicar los criterios de búsqueda descritos se recuperaron un total de 379 referencias: 186 $(49,1 \%)$ en Scopus, 165 (43,5\%) en Web of Science, 10 (2,6\%) en MEDLINE (vía PubMed), 9 (2,4\%) en Embase y 9 (2,4\%) en Cochrane Library.

Una vez depurados los 107 (28,2\%) registros repetidos, aplicar los criterios de inclusión y exclusión, y consultar los listados bibliográficos de los artículos seleccionados mediante búsqueda manual (figura 1), fue posible seleccionar 7 documentos (13-19) para su revisión y análisis crítico (tabla 1). Un artículo fue recogido de la bibliografía de los trabajos anteriormente seleccionados. 


\section{Figura 1 - Identificación y selección de estudios}

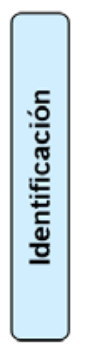

\section{Registros identificados a través de la búsqueda en bases de datos} $n=379$
Registros adicionales identificados a través de otras fuentes (búsqueda manual)

$$
n=1
$$

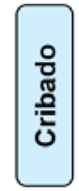

Registros repetidos

(Recuperado en más de una base de datos) $\mathrm{n}=107$
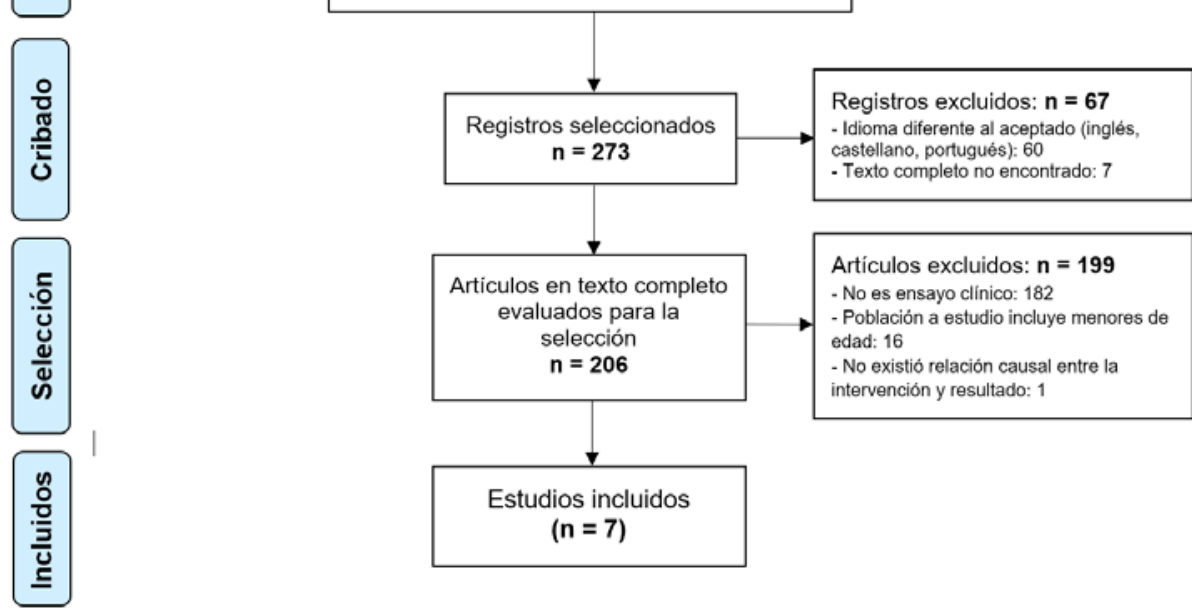
Tabla 1. Resumen de los estudios revisados sobre la calidad de vida de las personas adultas ingresadas en hospitalización domiciliaria con nutrición parenteral

\begin{tabular}{|c|c|c|c|c|c|c|c|c|c|}
\hline $\begin{array}{l}\text { Autor, año } \\
\text { PMID }\end{array}$ & Diseño & $\begin{array}{l}\text { Población } \\
\text { estudiada }\end{array}$ & Patología & País & $\begin{array}{l}\text { Periodo } \\
\text { del } \\
\text { estudio }\end{array}$ & $\begin{array}{l}\text { Tipo de } \\
\text { nutrición }\end{array}$ & $\begin{array}{l}\text { Forma y } \\
\text { frecuencia de } \\
\text { administración }\end{array}$ & $\begin{array}{l}\text { Método de } \\
\text { evaluación } \\
\text { de la } \\
\text { calidad de } \\
\text { vida }\end{array}$ & $\begin{array}{l}\text { Resultado } \\
\text { observado }\end{array}$ \\
\hline $\begin{array}{l}\text { Bohnert, et al. } \\
2018 \text { (13) }\end{array}$ & $\begin{array}{l}\text { Ensayo } \\
\text { clínico } \\
\text { aleatorizado } \\
\text { controlado }\end{array}$ & $\begin{array}{l}N=42 \\
\text { G1: Test } \\
n=21 \\
H / M= \\
66,7 \% / 33.3 \% \\
\text { Edad }=55,8 \\
\pm 15,1 \\
\text { G2: Control } \\
n=21 \\
\text { H/M }= \\
57,1 \% / 42,9 \% \\
\text { Edad }=58 \pm 13\end{array}$ & Malabsorción & Alemania & $\begin{array}{l}8 \\
\text { semanas; } \\
\text { entre } \\
02 / 2008 \text { y } \\
01 / 2014\end{array}$ & $\begin{array}{l}\text { Comparación } \\
\text { nutrición } \\
\text { parenteral } \\
\text { con AGPI* } \\
\text { n-3 y } \\
\text { nutrición } \\
\text { parenteral sin } \\
\text { AGPI n-3 }\end{array}$ & $\begin{array}{l}\text { A través } \\
\text { de catéter } \\
\text { venoso central } \\
\text { e infusión } \\
\text { continua } \\
\text { durante la } \\
\text { noche }\end{array}$ & $\begin{array}{l}\text { EORTC- } \\
\text { QLQ-C30 }\end{array}$ & $\begin{array}{l}\text { No hubo } \\
\text { asociación } \\
\text { entre los dos } \\
\text { grupos en } \\
\text { relación a la } \\
\text { calidad de } \\
\text { vida. }\end{array}$ \\
\hline $\begin{array}{l}\text { Hurt, et al. } \\
2017 \text { (14) }\end{array}$ & $\begin{array}{l}\text { Ensayo } \\
\text { clínico } \\
\text { aleatorizado } \\
\text { controlado }\end{array}$ & $\begin{array}{l}N=22 \\
G 1: \\
n=11 \\
H / M=5 / 6 \\
E d a d=58,8 \\
\pm 7,9 \\
G 2: \\
n=11 \\
H / M=3 / 8 \\
E d a d=55 \pm 21\end{array}$ & $\begin{array}{l}\text { Síndrome } \\
\text { de intestino } \\
\text { corto }\end{array}$ & EE. UU. & 6 meses. & No consta & No consta & $\begin{array}{l}\text { Escala } \\
\text { analógica } \\
\text { visual del } \\
0-10\end{array}$ & $\begin{array}{l}\text { No hubo } \\
\text { asociación } \\
\text { entre los dos } \\
\text { grupos en } \\
\text { relación a la } \\
\text { calidad de } \\
\text { vida. }\end{array}$ \\
\hline $\begin{array}{l}\text { Saqui, et al. } \\
2014(15)\end{array}$ & $\begin{array}{l}\text { Ensayo } \\
\text { clínico } \\
\text { aleatorizado } \\
\text { controlado }\end{array}$ & $\begin{array}{l}N=20 \\
H / M=5 / 15 \\
E d a d=52,8 \\
\pm 3,3\end{array}$ & $\begin{array}{l}\text { Síndrome } \\
\text { de intestino } \\
\text { corto }(50 \%) \text { y } \\
\text { malabsorción } \\
(25 \%)\end{array}$ & Canadá & 2 meses & No consta & $\begin{array}{l}\text { Bomba } \\
\text { estacionaria o } \\
\text { bomba portátil } \\
\text { e infusión } \\
\text { durante la } \\
\text { noche ( } 11,2 \pm \\
0,3 \text { horas) y } 4,3 \\
\pm 0,4 \text { días a la } \\
\text { semana }\end{array}$ & SF-36.v2 & $\begin{array}{l}\text { El grupo } \\
\text { con bomba } \\
\text { portátil valoró } \\
\text { ser más feliz } \\
\text { y tener mayor } \\
\text { comodidad. }\end{array}$ \\
\hline $\begin{array}{l}\text { Jeppesen, et al. } \\
2013 \text { (16) }\end{array}$ & $\begin{array}{l}\text { Ensayo } \\
\text { clínico } \\
\text { aleatorizado } \\
\text { controlado }\end{array}$ & $\begin{array}{l}N=70 \\
G 1: \\
n=35 \\
\text { Edad }=51 \pm \\
12,7 \\
\text { G2: } \\
n=35 \\
E d a d=50 \pm 15\end{array}$ & $\begin{array}{l}\text { Síndrome } \\
\text { intestino } \\
\text { corto con } \\
\text { fracaso } \\
\text { intestinal }\end{array}$ & $\begin{array}{l}\text { No } \\
\text { consta }\end{array}$ & $\begin{array}{l}24 \\
\text { semanas; } \\
\text { entre } \\
11 / 2008 \text { y } \\
01 / 2011\end{array}$ & $\begin{array}{l}\text { Soporte } \\
\text { parenteral } \\
\text { con } \\
\text { teduglutida } \\
\text { y soporte } \\
\text { parenteral } \\
\text { con placebo. }\end{array}$ & $\begin{array}{l}\text { Se administró } \\
\text { una vez al } \\
\text { día mediante } \\
\text { una inyección } \\
\text { subcutánea en } \\
\text { el abdomen. }\end{array}$ & SBS-QoL & $\begin{array}{l}\text { El grupo } \\
\text { con soporte } \\
\text { parenteral } \\
\text { con } \\
\text { teduglutida } \\
\text { mostró mayor } \\
\text { calidad de } \\
\text { vida. }\end{array}$ \\
\hline
\end{tabular}




\begin{tabular}{|c|c|c|c|c|c|c|c|c|c|}
\hline $\begin{array}{l}\text { Autor, año } \\
\text { PMID }\end{array}$ & Diseño & $\begin{array}{l}\text { Población } \\
\text { estudiada }\end{array}$ & Patología & País & $\begin{array}{l}\text { Periodo } \\
\text { del } \\
\text { estudio }\end{array}$ & $\begin{array}{l}\text { Tipo de } \\
\text { nutrición }\end{array}$ & $\begin{array}{l}\text { Forma y } \\
\text { frecuencia de } \\
\text { administración }\end{array}$ & $\begin{array}{l}\text { Método de } \\
\text { evaluación } \\
\text { de la } \\
\text { calidad de } \\
\text { vida }\end{array}$ & $\begin{array}{l}\text { Resultado } \\
\text { observado }\end{array}$ \\
\hline $\begin{array}{l}\text { Culkin, et al. } \\
2008 \text { (17) }\end{array}$ & $\begin{array}{l}\text { Ensayo } \\
\text { clínico } \\
\text { aleatorizado } \\
\text { controlado }\end{array}$ & $\begin{array}{l}\mathrm{N}=22 \\
\mathrm{G} 1: \text { Glutamina } \\
\mathrm{n}=11 \\
\mathrm{H} / \mathrm{M}=5 / 6 \\
\text { Edad }=51,5 \\
\pm 12,1 \\
\mathrm{G} 2: \text { Estándar } \\
\mathrm{n}=11 \\
\mathrm{H} / \mathrm{M}=4 / 7 \\
\mathrm{Edad}=55,5 \\
\pm 12,8\end{array}$ & $\begin{array}{l}\text { Enfermedad } \\
\text { de Crohn, } \\
\text { infarto } \\
\text { mesentérico } \\
\text { y otros }\end{array}$ & $\begin{array}{l}\text { Reino } \\
\text { Unido }\end{array}$ & $\begin{array}{l}6 \text { meses o } \\
1 \text { año }\end{array}$ & $\begin{array}{l}\text { Comparación } \\
\text { nutrición } \\
\text { parenteral } \\
\text { con } \\
\text { glutamina } \\
\text { y nutrición } \\
\text { parenteral } \\
\text { estándar }\end{array}$ & $\begin{array}{l}\text { A través de } \\
\text { catéter durante } \\
6 \text { días a la } \\
\text { semana }\end{array}$ & $\begin{array}{l}\text { SF-36 y } \\
\text { EuroQol }\end{array}$ & $\begin{array}{l}\text { No hubo } \\
\text { diferencias } \\
\text { significativas } \\
\text { respecto a } \\
\text { la calidad de } \\
\text { vida, entre } \\
\text { el grupo con } \\
\text { glutamina y el } \\
\text { control. }\end{array}$ \\
\hline $\begin{array}{l}\text { Chambers, et al. } \\
2006 \text { (18) }\end{array}$ & $\begin{array}{l}\text { Ensayo } \\
\text { clínico } \\
\text { aleatorizado } \\
\text { controlado }\end{array}$ & $\begin{array}{l}\mathrm{N}=30 \\
\mathrm{G} 1: \\
\mathrm{n}=15 \\
\mathrm{H} / \mathrm{M}=8 / 7 \\
\mathrm{Edad}=42,1 \\
\mathrm{G} 2: \\
\mathrm{n}=15 \\
\mathrm{H} / \mathrm{M}=5 / 10 \\
\mathrm{Edad}=37,5\end{array}$ & $\begin{array}{l}\text { Intestino } \\
\text { corto } \\
\text { debido a } \\
\text { enfermedad } \\
\text { de Crohn y } \\
\text { enfermedad } \\
\text { vascular } \\
\text { mesentérica. }\end{array}$ & $\begin{array}{l}\text { Reino } \\
\text { Unido }\end{array}$ & $\begin{array}{l}12 \text { meses; } \\
\text { entre } \\
03 / 2001 \text { y } \\
06 / 2003\end{array}$ & No consta & No consta & $\begin{array}{l}\text { SF-36 y } \\
\text { EQ5D }\end{array}$ & $\begin{array}{l}\text { No hubo } \\
\text { asociación } \\
\text { entre los } \\
\text { dos grupos } \\
\text { respecto a } \\
\text { la calidad de } \\
\text { vida. }\end{array}$ \\
\hline $\begin{array}{l}\text { Smith, et al. } \\
2003(19)\end{array}$ & $\begin{array}{l}\text { Ensayo } \\
\text { clínico } \\
\text { aleatorizado } \\
\text { controlado }\end{array}$ & $\begin{array}{l}\mathrm{N}=73 \\
\mathrm{G} 1: \\
\text { Experimental } \\
\mathrm{n}=35 \\
\mathrm{H} / \mathrm{M}= \\
30,8 \% / 69,2 \% \\
\text { Edad }=48,42 \\
\pm 12,4 \\
\mathrm{G} 2 \text { : Control } \\
n=38 \\
\mathrm{H} / \mathrm{M}= \\
32,4 \% / 67,6 \% \\
\text { Edad }=49,83 \pm \\
16,67\end{array}$ & $\begin{array}{l}\text { Síndrome } \\
\text { de intestino } \\
\text { corto no } \\
\text { maligno }\end{array}$ & EE. UU. & 18 meses & No consta & No consta & $\begin{array}{l}\text { Quality of } \\
\text { Life Index }\end{array}$ & $\begin{array}{l}\text { El grupo } \\
\text { experimental } \\
\text { presentó } \\
\text { mayores } \\
\text { mejoras en } \\
\text { la calidad } \\
\text { de vida } \\
\text { debido a la } \\
\text { disminución } \\
\text { de } \\
\text { infecciones } \\
\text { por catéter. }\end{array}$ \\
\hline
\end{tabular}

El acuerdo sobre la pertinencia de los estudios seleccionados, entre los dos evaluadores, calculado mediante el índice Kappa fue del 100\%.

Los artículos elegidos presentaron una obsolescencia, según el Índice de Burton Kebler, igual a 7 años, con un Índice de Price del 28,6\%.

Al evaluar la calidad de los artículos seleccionados para la revisión, mediante el cuestionario CONSORT, las puntuaciones oscilaron entre un mínimo de 12,5 (sobre 24 ítems) y un máximo de 20,5 (sobre 24 items) con mediana igual a 17,0 (tabla 2). 
Según los criterios de la U. S. Agency for Healthcare Research and Quality (AHRQ) esta revisión presentó evidencia lb (procede de al menos un ensayo clínico aleatorizado) con grado de recomendación B (basada en estudios de las categorías Ib, Ila y llb).

Todos los revisados fueron ensayos clínicos controlados aleatorizados; la filiación fue en 2 de ellos británica $(17,18)$ y en otros 2 estadounidense $(14,19)$. Alemania y Canadá presentaron 1 trabajo $(13,15)$. En uno de los ensayos no figuraba el país de filiación (16). Todos los artículos estaban redactados en inglés.

La población incluida, toda ella adulta, superó la media de 48 años en la mayoría de los estudios, excepto el trabajo de Chambers et al. (18), en el cual estaba comprendida entre los 37,5 y 42,1 años, y presentó un adecuado cociente hombre/mujer; destacar el trabajo de Saqui et al. (15), donde el $75 \%$ de la población eran mujeres y la ausencia de la especificación por sexos en el estudio de Jeppesen et al. (16), siendo este el trabajo con mayor número de personas ( $N=70)$.

La patología predominante fue el síndrome de intestino corto (SIC), presente en 6 publicaciones (86\%) (14-19).

El periodo de seguimiento de los estudios revisados osciló entre un mínimo de 8 semanas (13) y un máximo de 18 meses (19).

\section{Tipo de nutrición parenteral: forma y frecuencia de administración}

En 2 de los ensayos la nutrición contenía algún fármaco: teduglutida (16) y glutamina (17). En el estudio de Bohnert et al. (13), se adicionó, a la nutrición parenteral, ácidos grasos poliinsaturados n-3.

En cuanto a la forma y frecuencia de administración, se informó en 4 estudios (13,15-17): siendo la administración vía catéter la elegida en 2 de ellos $(13,17)$. En 3 de los 7 estudios $(14,18,19)$, no constaba la forma de administración utilizada.

\section{Evaluación de la calidad de vida}

La evaluación de la calidad de vida fue analizada mediante cuestionarios, siendo el genérico SF-36 (Short Form-36 Healt) el utilizado en 3 estudios $(15,17,18)$. En 2 ensayos $(17,18)$, fue empleado, además, un cuestionario genérico, el EuroQoL-5D (Euro Quality of Life 5 Dimencions). Hurt et al. (14), utilizaron una escala analógica visual genérica VAS (Visual Analogue Scale). El ensayo de Smith et al. (19) empleó el Quality of life index, un cuestionario utilizado en pacientes con nutrición parenteral domiciliaria, hemodiálisis o pacientes tratados por cáncer.

Bohnert et al. (13), emplearon un cuestionario específico para las neoplasias, el EORTC-QLQC30 (European Organization for Research and Treatment of Cancer Quality of Life) y en el trabajo de Jeppesen et al. (16) se utilizó el específico para el síndrome de intestino corto, SBS-QoL (Short Bowel Syndrome-Quality of Life).

\section{Resultados relacionados con la calidad de vida}

En general, los resultados obtenidos en los distintos ensayos revisados, no demostraron asociación entre los grupos a estudio. En 3 de ellos se lograron resultados con diferencias significativas $(15,16,19)$. En la investigación de Saqui et al. (15), se vio que el grupo que llevaba bomba portátil, obtuvo mayor puntuación en la calidad de vida; en la de Jeppesen et al. (16), donde el grupo con soporte parenteral con teduglutida mostró mayor calidad de vida y el trabajo de Smith et al. (19), 
donde se observó mayor calidad de vida en el grupo que había tenido menor número de infecciones motivadas por el catéter.

\section{DISCUSIÓN}

En este estudio se comprobó el escaso número de ensayos clínicos, existentes en las bases de datos bibliográficas internacionales, que hubieran estudiado la calidad de vida en pacientes ingresados en $\mathrm{HaD}$ con nutrición parenteral. Además, de los seleccionados, únicamente los ensayos de Saqui et al. (15), de Smith et al. (19) y de Jeppesen et al. (16), ofrecieron asociación significativa en relación a la calidad de vida.

El análisis de la actualidad/obsolescencia de los estudios incluidos demostró la plena vigencia del tema estudiado ya que los datos calculados mostraron menor obsolescencia que los datos usuales en el ámbito de los estudios sobre hospitalización domiciliaria (20,21).

Los resultados alcanzados en la puntuación CONSORT, muestran que la calidad de los estudios evaluados, en la mayoría de los casos, fue adecuada. Aunque, equivalente a la observada en otras revisiones similares $(4,7)$. De todos modos, no se eliminó ningún estudio para no limitar información a los posibles lectores de esta revisión. Aun así, sorprende la baja puntuación conseguida por los trabajos de Saqui et al. (15), Hurt et al. (14) o Chambers et al. (18).

El diseño de los estudios revisados mostró una buena evidencia según las recomendaciones de la U. S. Agency for Healthcare Research and Quality (AHRQ) (12). El ceñir la revisión a ensayos clínicos se debió a la búsqueda de una consistente relación causa-efecto, ya que el vínculo existente entre el estado nutricional y la calidad de vida se está convirtiendo en una cuestión importante no sólo en los pacientes oncológicos, sino también en otras patologías e intervenciones $(2,4)$.

Era predecible la filiación anglófona de una mayoría de artículos y que estuvieran redactados, principalmente, en lengua inglesa. Este idioma es el elegido para la publicación de la mayoría de los artículos ya que hacerlo en otra lengua distinta resulta negativo para la visibilidad y análisis de citas. Asimismo, el número de revistas anglófonas contenidas en las principales bases de datos bibliográficas es muy elevado y publicar en ellas facilita la citación (21,22).

La población incluida en los estudios presentó edades inferiores a las observadas en anteriores estudios relacionados con la población ingresada en las unidades de HaD, que suelen ser personas mayores con patologías crónicas o terminales $(6,23)$. Ahora, una revisión sistemática sobre las consecuencias de la nutrición parenteral domiciliaria en adultos con síndrome de intestino corto (24), patología predominante en este estudio, también incluyó adultos con una edad similar a la que se observó en esta revisión. Asimismo, el bajo número de personas adultas reclutadas en las investigaciones era predecible. Este fracaso intestinal está catalogado como enfermedad rara por su baja prevalencia lo que dificulta el poder disponer de cohortes suficientes para realizar ensayos clínicos robustos (24).

En cuanto al período de seguimiento resultó adecuado para valorar los resultados de la intervención (efectos de la nutrición parenteral), requisito que cumplieron la mayoría de los estudios seleccionados. Se considera necesario un periodo de varias semanas para poder valorar los resultados (4).

Los resultados de esta revisión muestran que para evaluar el impacto de la intervención nutricional sobre la calidad de vida de los pacientes paliativos es práctica habitual utilizar cuestionarios validados, como ocurre en los estudios incluidos en esta revisión, a excepción del trabajo de Hurt et al. (14) que usa una escala analógica visual. Hay que tener en cuenta que la utilización de herramientas genéricas requeriría mayores tamaños poblacionales para poder conseguir resultados 
contrastables y en la mayoría de las ocasiones estos cuestionarios se ven afectados por problemas externos $(2,25)$.

En relación a la medición de la calidad de vida relacionada con el estado nutricional hay que tener en cuenta que se verá influenciada por la percepción del paciente, el impacto de la enfermedad, el tratamiento, las expectativas y el bienestar. Por tanto, deben buscarse herramientas validadas que permitan minimizar cualquier tipo de subjetividad del paciente $(2,4)$. En esta revisión se ha constatado el uso de estos cuestionarios. De todos modos, el uso de herramientas validadas no garantiza la obtención de resultados generalizables, como se observó en el estudio de Baldwin et al. (26).

El estudio incluido de Saqui et al., el grupo con bomba portátil valoró ser más feliz y tener mayor comodidad, con mejor calidad de vida. Los resultados que relacionaban la calidad de vida con el uso de una bomba portátil se debían principalmente a que las personas que cumplen criterios para recibir NPD también agradecen la independencia. La bomba portátil permite a estas personas, que ya sufrieron un cambio importante en su estilo de vida, lograr la libertad deseada y motiva la aceptación de la NPD (27). Esta conformidad es realmente importante, pues el tratamiento puede durar toda la vida e impone severas restricciones en el quehacer diario (25). Afortunadamente, desde hace ya tiempo, hay muchas bombas portátiles compactas disponibles que hacen que la terapia en el hogar sea más aceptable (24).

El trabajo de Jeppesen et al. (16), mostró que el grupo con soporte parenteral con teduglutida tuvo una mayor calidad de vida. El beneficio del uso de la teduglutida para el equilibrio de líquidos y la absorción de monosacáridos ya había sido comunicado en un trabajo anterior, de este mismo autor, donde se afirmaba que este principio era intestinotrófico y sugería efectos pro-absorbentes en pacientes con SIC (28). En el mismo sentido, Vipperla y O'Keefe (29), señalaron que este fármaco, si bien debía ser mejor evaluado para la práctica clínica, ofrecía un nuevo enfoque específico para el manejo de la insuficiencia intestinal asociada al SIC. Kochar y Herfarth (30), en una revisión publicada en 2018, ultimaron que, por los datos disponibles, aunque limitados debido al pequeño número de pacientes en los estudios realizados hasta ahora, la teduglutida parece ser segura para el uso en pacientes con insuficiencia intestinal que dependen del soporte parenteral.

Smith et al. (19), observaron que el grupo experimental presentó mejor calidad de vida debido a la disminución de infecciones por catéter. Esta situación había sido ya advertida, recientemente, por Skelton-Dudley et al. (31), que recomendaban minimizar el daño relacionado con el catéter (incluida las infecciones), ya que reducían la resistencia a los antibióticos y mejoraban la satisfacción y la calidad de vida general de los pacientes.

En general, y en relación a la intervención, la NPD, ya existía evidencia sobre un impacto significativo, positivo, en la calidad de vida. Su implantación presentó resultados generalmente buenos y la supervivencia estuvo determinada, principalmente, por la enfermedad subyacente $(27,32)$.

Posibles limitaciones al estudio: La principal limitación sería el escaso número de estudios recuperados que impidió tener una clara evidencia entre la intervención nutricional y la mejora, o no, de la calidad de vida.

La diferencia entre el número de artículos conseguidos en la búsqueda bibliográfica y los realmente válidos se debió a las bases de datos Scopus y Web of Science, las cuales no disponen de Thesaurus, por lo que no permiten el uso de Descriptores para la búsqueda precisa de los ensayos, aumentando la aparición de "ruido" en los documentos recuperados tras la búsqueda.

Otra limitación de la presente revisión fue no poder recuperar el texto completo de algunos artículos, siendo los principales motivos no estar digitalizados en la web de la revista, no aparecer en las principales colecciones de revistas e incluso no poder ser recuperado a través de la red de bibliotecas universitarias. 
En base a lo anteriormente expuesto, podemos concluir que: el incremento de la calidad de vida de los enfermos con NPD está directamente relacionado con la del estado y soporte nutricional de los mismos. Se observó una mejor calidad de vida en los pacientes con bomba portátil y en los que se administró teduglutida. El adecuado manejo del catéter y la consecuente disminución de las infecciones también contribuyó a la mejora de la calidad de vida de las personas con NPD.

\section{BIBLIOGRAFÍA}

1. Hendry F, McVittie C. Is quality of life a healthy concept? Measuring and understanding life experiences of older people. Qual Health Res. 2004;14(7):961-75. DOI: 10.1177/1049732304266738; PMID: 15296666

2. Wanden-Berghe C, Sanz-Valero J, Escribà-Agüir V, Castelló-Botia I, Guardiola-Wanden-Berghe R, Red de Malnutrición en Iberoamérica - Ciencia y Tecnología para Desarrollo (Red Mel CYTED). Evaluation of quality of life related to nutritional status. Br J Nutr. 2009;101(7):950-60. DOI: 10.1017/S0007114508207178; PMID: 19183508

3. Pearson JM, Schlettwein-Gsell D, Brzozowska A, van Staveren WA, Bjørnsbo K. Life style characteristics associated with nutritional risk in elderly subjects aged 80-85 years. J Nutr Health Aging. 2001;5(4):278-83. PMID: 11753495

4. Aracil-Lavado E, Wanden-Berghe C, Sanz-Valero J. Evaluación de la calidad de vida según el estado nutricional del paciente paliativo adulto: revisión sistemática. Hosp Domic. 2017;1(4):199210. DOI: 10.22585/hospdomic.v1i4.27

5. Bourdel-Marchasson I, Joseph PA, Dehail P, Biran M, Faux P, Rainfray M, et al. Functional and metabolic early changes in calf muscle occurring during nutritional repletion in malnourished elderly patients. Am J Clin Nutr. 2001;73(4):832-8. DOI: 10.1093/ajcn/73.4.832; PMID: 11273861

6. Estrada Cuxart O, Massa Domínguez B, Ponce González MÁ, Mirón Rubio M, Torres Corts A, Mujal Martínez A, et al. Proyecto HAD 2020: una propuesta para consolidar la hospitalización a domicilio en España. Hosp Domic. 2017;1(2):93-117. DOI: 10.22585/hospdomic.v1i2.13

7. Álvarez Velásquez S, Sanz Valero J. Ventajas de la quimioterapia domiciliaria en los enfermos adultos con neoplasias: revisión sistemática. Hosp Domic. 2020;4(1):23-39. DOI: 10.22585/hospdomic.v4i1.98

8. Wanden-Berghe C, Pereira Cunill JL, Cuerda Compes C, Ramos Boluda E, Maiz Jiménez MI, Gómez Candela C, et al. Nutrición parenteral domiciliaria en España 2017: Informe del Grupo de Nutrición Artificial Domiciliaria y Ambulatoria NADYA. Nutr Hosp. 2018;35(6):1491-6. DOI: 10.20960/nh.2364; PMID: 30525864

9. Wanden-Berghe C, Nolasco A, Planas M, Sanz-Valero J, Rodríguez T, Cuerda C, et al. Healthrelated quality of life according to the main caregiver in patients with home nutritional support. Med Clin (Barc). 2008;131(8):281-4. DOI: 10.1016/s0025-7753(08)72258-9; PMID: 18803920

10. Mateo-Lobo R, Riveiro J, Vega-Piñero B, Botella-Carretero JI. Infectious Complications in Home Parenteral Nutrition: A Systematic Review and Meta-Analysis Comparing Peripherally-Inserted Central Catheters with Other Central Catheters. Nutrients. 2019;11(9):pii: E2083. DOI: 10.3390/ nu11092083; PMID: 31487777 
11. Cobos-Carbó A, Augustovski F. Declaración CONSORT 2010: actualización de la lista de comprobación para informar ensayos clínicos aleatorizados de grupos paralelos. Med Clin (Barc). 2011;137(5):213-5. DOI: 10.1016/j.medcli.2010.09.034; PMID: 21239025

12. Berkman ND, Lohr KN, Ansari M, McDonagh M, Balk E, Whitlock E, et al. Grading the Strength of a Body of Evidence When Assessing Health Care Interventions for the Effective Health Care Program of the Agency for Healthcare Research and Quality: An Update. In: Methods Guide for Effectiveness and Comparative Effectiveness Reviews. Rockville (MD): Agency for Healthcare Research and Quality (US); 2008. PMID: 24404627

13. Bohnert H, Maurer M, Calder PC, Pratschke J, Thul P, Müller V. Efficacy of a long-term home parenteral nutrition regimen containing fish oil-derived n-3 polyunsaturated fatty acids: a single-centre, randomized, double blind study. Nutr J. 2018;17(1):113. DOI: 10.1186/s12937-0180419-x; PMID: 30501620

14. Hurt RT, Vallumsetla N, Edakkanambeth Varayil J, Bonnes SL, Nanda S, Nadeau J, et al. Pilot Study Comparing 2 Oral Rehydration Solutions in Patients with Short Bowel Syndrome Receiving Home Parenteral Nutrition: A Prospective Double-Blind Randomized Controlled Trial. Nutr Clin Pract. 2017;32(6):814-9. DOI: 10.1177/0884533617714975; PMID: 28662613

15. Saqui O, Fernandes G, Allard JP. Quality of life analysis during transition from stationary to portable infusion pump in home parenteral nutrition patients: a Canadian experience. Nutr Clin Pract. 2014;29(1):131-41. DOI: 10.1177/0884533613516129; PMID: 24347531

16. Jeppesen PB, Pertkiewicz M, Forbes A, Pironi L, Gabe SM, Joly F, et al. Quality of life in patients with short bowel syndrome treated with the new glucagon-like peptide-2 analogue teduglutide--analyses from a randomised, placebo-controlled study. Clin Nutr. 2013;32(5):713-21. DOI: 10.1016/j.cInu.2013.03.016; PMID: 23587733

17. Culkin A, Gabe SM, Bjarnason I, Grimble G, Madden AM, Forbes A. A double-blind, randomized, controlled crossover trial of glutamine supplementation in home parenteral nutrition. Eur $\mathrm{J}$ Clin Nutr. 2008;62(5):575-83. DOI: 10.1038/sj.ejcn.1602754; PMID: 17440526

18. Chambers A, Hennessy E, Powell-Tuck J. Longitudinal trends in quality of life after starting home parenteral nutrition: a randomised controlled study of telemedicine. Clin Nutr. 2006;25(3):50514. DOI: 10.1016/j.cInu.2006.01.001; PMID: 16698138

19. Smith CE, Curtas S, Kleinbeck SVM, Werkowitch M, Mosier M, Seidner DL, et al. Clinical trial of interactive and videotaped educational interventions reduce infection, reactive depression, and rehospitalizations for sepsis in patients on home parenteral nutrition. JPEN J Parenter Enteral Nutr. 2003;27(2):137-45. DOI: 10.1177/0148607103027002137; PMID: 12665170

20. Sanz-Valero J, Wanden-Berghe C. Análisis bibliométrico de la producción científica, indizada en MEDLINE, sobre los servicios de salud proporcionados por las unidades de hospitalización a domicilio. Hosp Domic. 2017;1(1):21-34. DOI: 10.22585/hospdomic.v1i1.3

21. Oller-Arlandis VE, Sanz Valero J, Wanden-Berghe C. Análisis bibliométrico de los ensayos clínicos, indizados hasta el año 2018, en la base bibliográfica Cochrane Library realizados en el ámbito de la hospitalización a domicilio. Hosp Domic. 2019;3(4):255-68. DOI: 10.22585/hospdomic.v3i4.88

22. Domingo-Pueyo A. Análisis bibliométrico de la producción científica española, indizada en MEDLINE, sobre servicios de atención a domicilio provisto por hospital. Hosp Domic. 2017;1(3):14151. DOI: 10.22585/hospdomic.v1i3.24 
23. Mas MÀ, Santaeugènia S. Hospitalización domiciliaria en el paciente anciano: revisión de la evidencia y oportunidades de la geriatría. Rev Esp Geriatr Gerontol. 2015;50(1):26-34. DOI: 10.1016/j.regg.2014.04.003

24. Campos Cañuelo D, Sanz-Valero J, Wanden-Berghe C. Consecuencias de la nutrición parenteral domiciliaria en adultos con síndrome de intestino corto: revisión exploratoria. Hosp Domic. 2019;3(2):149-62. DOI: 10.22585/hospdomic.v3i2.60

25. Baxter JP, Fayers PM, McKinlay AW. A review of the instruments used to assess the quality of life of adult patients with chronic intestinal failure receiving parenteral nutrition at home. $\mathrm{Br} \mathrm{J}$ Nutr. 2005;94(5):633-8. DOI: 10.1079/bjn20051533; PMID: 16277762

26. Baldwin C, Spiro A, McGough C, Norman AR, Gillbanks A, Thomas K, et al. Simple nutritional intervention in patients with advanced cancers of the gastrointestinal tract, non-small cell lung cancers or mesothelioma and weight loss receiving chemotherapy: a randomised controlled trial. J Hum Nutr Diet. 2011;24(5):431-40. DOI: 10.1111/j.1365-277X.2011.01189.x; PMID: 21733143

27. Boutin J, Hagan E. Patients' preference regarding portable pumps. J Intraven Nurs. 1992;15(4):230-2. PMID: 1500992

28. Jeppesen PB, Gilroy R, Pertkiewicz M, Allard JP, Messing B, O'Keefe SJ. Randomised placebo-controlled trial of teduglutide in reducing parenteral nutrition and/or intravenous fluid requirements in patients with short bowel syndrome. Gut. 2011;60(7):902-14. DOI: 10.1136/ gut.2010.218271; PMID: 21317170

29. Vipperla K, O'Keefe SJ. Study of teduglutide effectiveness in parenteral nutrition-dependent short-bowel syndrome subjects. Expert Rev Gastroenterol Hepatol. 2013;7(8):683-7. DOI: 10.1586/17474124.2013.842894; PMID: 24134154

30. Kochar B, Herfarth HH. Teduglutide for the Treatment of Short Bowel Syndrome - A Safety EvaIuation. Expert Opin Drug Saf. 2018;17(7):733-739. DOI: 10.1080/14740338.2018.1483332; PMID: 29848084

31. Skelton-Dudley F, Doan J, Suda K, Holmes SA, Evans C, Trautner B. Spinal Cord Injury Creates Unique Challenges in Diagnosis and Management of Catheter-Associated Urinary Tract Infection. Top Spinal Cord Inj Rehabil. 2019;25(4):331-9. DOI: 10.1310/sci2504-331; PMID: 31844385

32. Ravasco P, Monteiro-Grillo I, Marques Vidal P, Camilo ME. Impact of nutrition on outcome: a prospective randomized controlled trial in patients with head and neck cancer undergoing radiotherapy. Head Neck. 2005;27(8):659-68. DOI: 10.1002/hed.20221; PMID: 15920748 


\section{Tabla 2. Análisis de la calidad metodológica de los estudios a través de los 25 items de valoración de la guía CONSORT}

\begin{tabular}{|c|c|c|c|c|c|c|c|c|c|c|c|c|c|c|c|c|c|c|c|c|c|c|c|c|c|c|c|}
\hline & 1 & 2 & 3 & 4 & 5 & 6 & 7 & 8 & 9 & 10 & 11 & 12 & 13 & 14 & 15 & 16 & 17 & 18 & 19 & 20 & 21 & 22 & 23 & 24 & 25 & Total & Total (\%) \\
\hline Bohnert, et al. (13) & 0.5 & 1 & 1 & 1 & 1 & 0.5 & 1 & 1 & 1 & 0 & 0.5 & 1 & 1 & 0 & 1 & 1 & 1 & NA & 1 & 1 & 1 & 1 & 1 & 1 & 1 & 20.5 & $85 \%$ \\
\hline Hurt, et al. (14) & 1 & 1 & 0.5 & 1 & 1 & 0.5 & 0 & 0 & 0 & 0 & 0 & 1 & 1 & 0.5 & 1 & 1 & 1 & NA & 1 & 1 & 0 & 1 & 0 & 0 & 1 & 14.5 & $60 \%$ \\
\hline Saqui, et al. (15) & 0.5 & 1 & 0.5 & 0.5 & 1 & 1 & 0 & 1 & 0 & 0 & 0 & 1 & 1 & 0 & 1 & 0 & 1 & NA & 0 & 1 & 1 & 1 & 0 & 0 & 0 & 12.5 & $52 \%$ \\
\hline Jeppesen, et al. (16) & 1 & 1 & 1 & 0.5 & 1 & 1 & 0.5 & 0.5 & 0 & 0 & 0 & 1 & 1 & 0.5 & 1 & 1 & 1 & NA & 1 & 1 & 1 & 1 & 0 & 0 & 1 & 17 & $71 \%$ \\
\hline Culkin, et al. (17) & 1 & 1 & 1 & 1 & 1 & 1 & 0.5 & 0.5 & 0 & 1 & 0.5 & 1 & 1 & 0 & 1 & 1 & 1 & NA & 1 & 1 & 1 & 1 & 0 & 0 & 1 & 18.5 & $77 \%$ \\
\hline Chambers, et al. (18) & 0.5 & 1 & 0 & 1 & 1 & 0.5 & 0.5 & 0.5 & 0 & 0 & 0 & 1 & 1 & 0.5 & 1 & 1 & 1 & NA & 1 & 1 & 0 & 1 & 0 & 0 & 1 & 14.5 & $60 \%$ \\
\hline Smith, et al. (19) & 1 & 1 & 1 & 1 & 1 & 1 & 1 & 0 & 0 & 0 & 0.5 & 1 & 0.5 & 0 & 1 & 1 & 1 & NA & 1 & 1 & 1 & 1 & 1 & 1 & 1 & 19 & $79 \%$ \\
\hline
\end{tabular}

